\title{
Route Repair through Route Discovey in MANET
}

\author{
Mehajabeen Fatima ${ }^{1}$, Roopam Gupta ${ }^{2}$ and T.K. Bandhopadhyay ${ }^{3}$ \\ RGPV, Bhopal ${ }^{1,2}$, India; BIST, Bhopal, India ${ }^{3}$ \\ mehajabeen.fatima@gmail.com ${ }^{1}$,roopam_1710@yahoo.co.in, ${ }^{2}$ \\ tushar_bando@gmail.com ${ }^{3}$
}

\begin{abstract}
Mobile ad hoc networks (MANETs) are mobility sensitive and battery power constraint network. The link breaks frequently, hampers the continuity of data transmission. Most of the research work is targeted to find a feasible route from a source to a destination without considering its link breakage possibilities and its after effects. So proper route management is required. Thus, route discovery using cross layer approach routing protocol with Fuzzy logic (RDCLRP-FUZZY) is proposed in this paper. The route is refurbished through route discovery using adaptive hello warning message (HWM). The proposed scheme is investigated for mobility and traffic. Simulations are run on qualnet 5.0. Results are compared with basic AODV. It is illustrated that the route failure is reduced by $90 \%$ which is most important aspect for real time communication. The reduction of route failure improves the performance of the network. The novel part of the proposed work is route maintenance through route discovery. This reduces route failure frequency and flooding in the network.
\end{abstract}

Keywords: MANET, cross layer approach, critical node, route repair, route discovery, Fuzzy, Hello Warning message

\section{Introduction}

MANET is featured by mobility, dynamic topology, multi-hop communication, limited resources and limited security [1]. The nodes have limited battery and routing capabilities [2]. Now a days Email and Web browsing is common and widespread. The use of devices such as smart phones, tablets etc. offer the ease and convenience of internet applications. Mobile adhoc network should also include such features. Inevitably user expectations also rise in terms of higher data rates, instant internet connectivity, online games, chat, medical facility for health and variety of applications. If it is possible to provide all above apps in MANET then it will definitely increases the demand and traffic in future. To cope with high mobility and high traffic, routing protocol must be able to adapt the changes. [3] S. Iyer et.al., 2002 analyzed that the proactive protocols perform well for heavy traffic load. [4] Xiaoxia Huang et.al., 2009 discussed that the reactive protocols perform well when the network load is moderate. The reactive routing protocol performance degrades for high traffic. It is also difficult to maintain uninterrupted communication of data with the increase in traffic, mobility, power consumption. When a link failure occurs, the TCP sender reacts as if congestion was the cause. It reduces its congestion window. This results in packet loss and bandwidth loss unnecessarily. This may lead to delay in communication and poses serious concern towards the real time applications[3].

The route failure is the main challenge in incorporation of above apps in MANET. The route breakdown is directly proportional to velocity of a node and traffic or battery power consumption $(\mathrm{BpC})$. Apart from this, the hierarchical nature of TCPUIP is another problem. TCPUIP architecture follows hierarchy and only adjacent layer can communicate 
with each other. The dynamics of MANET can't be handled with TCPUP architecture [5]. So cross layer architecture can be used. In cross layer design, all layers can communicate with each other resulting in quick and correct decision [6]. The careful exploitation of protocol stack interactions that cross the normal layer boundaries lead to more efficient performance of the network. The cross layer approach can be used to reduce the route failure rate.

Many researchers address the route failure issues. T. Goff et.al. in 2001 proposed a preemptive routing, aims to reduce the possibility of route breakege [7]. Wen-Hsin Yang et.al., 2010 defined the handover schemes instead of route repair [8]. As suggested by researchers, it is found that the preemptive route repair is better option and this motivated us to investigate into this field. An alternative path is sought only after an active path fails. The cost of detecting a path failure is high. Thus, it is required to design the strong route repair scheme so that performance of routing protocol can be improved [9]. It is also required to consider all parameters of route breakage to deduce the results towards real scenario.

Overview of AODV is given in Part 2. Part 3 explains proposed algorithm named as RDCLRP-FUZZY. Part 4 has given parameter calculation, Part 5 discussed results with graphs and then the conclusion is drawn in part 6.

\section{Overview of AODV}

AODV is an IETF standardizing and most popular reactive routing protocol designed by Charles E. Perkins and Elizabeth M. Royer. AODV operation is divided into two parts (a) Route establishment and (b) Route maintenance. Route establishment is done using Route Request message, RREQ and Route Reply message, RREP. Route maiantenance is done using Hello Message (HM) and Route Error message (RERR) [10]. Whenever a node wants to communicate, source checks its cache for the availability of route. If route is not available then source determines a route to a destination node through the RREQ message. The source node broadcasts a RREQ message to its neighboring nodes which in turn broadcast the message to their neighbors. Each node stores latest forwarded route requests in the register of route request [11]. When a node receiving the request either knows of a fresh enough route to the destination or is itself the destination, the node generates a RREP message and sends this message along the reverse path back towards the originating node $[11,12]$. Then the path is established and acts as active path.

Each active node periodically sends HM to its precursors [13]. If a node receives no messages from some outgoing node for some fixed period of time then that node is presumed to be no longer reachable. Then a node generates a RERR message. The node sends the RERR to each of its precursors. Then the source reinitiates the process of finding new route.

As soon as a node receives RREQ, start transmitting HM and maintains network connectivity. According to Ian Chakeres et. al in 2002 [13] and C. Perkins et. al. in 2003 [14], any broadcast control message serves as a HM determines network connectivity. It indicates the presence of a neighbor. If a node does not send any broadcast control message within a specified interval, a HM is broadcast. In this manner most of the nodes broadcast HM in the network. AODV is simple in operation and performs well but not perfect so far. It still has many issues like Flooding for route discovery, Delay in finding of route, Routing loop, Sender found no route, Deletion of existing route, Routing Table, Route maintenance, Power management, Unidirectional link problem, Security like selfish node and malicious node etc. There are many proposals available in the literature to solve these problems. But these issues are still open, need to be amended. In this paper AODV is modified to provide strong route maintenance. 


\section{Proposed Algorithm}

\section{Route Discovery using Cross Layer Routing Protocol with Active Nodes Hello and Fuzzy Logic (RDCLRP-FUZZY):}

Route maintenance with high mobility, high traffic and excessive node battery consumption is the challenge of routing protocol. This results in poor management of network, reduces quality of service and destabilize a network. Route maintenance becomes complicated if traffic or mobility in the network increases. Alternate route finding for broken link will improve the network performance. An alternative path is sought only after an active path fails. The expense of detecting a path failure is high compared to packet delays since many retrials and retransmissions have to time out before a path is confirmed as dead.

The challenges of AODV and TCPUIP architecture motivate us to work for improvement of protocol using cross layer approach. To improve performance of network, AODV is modified. We propose a modified distance vector routing protocol. In the proposed algorithm, the route discovery procedure is same as in AODV. The route maintenance process during communication is modified. Preemptive route repair is used for this purpose. Preemptive route repair means, a route should be repaired on highest priority before a route breakage. The route is repaired locally in advance before route failure, overhead is reduced due to local broadcast of maintenance packet in small area of network. Thus, some alteration have been proposed in the existing AODV routing protocol.

- Active precursor node IP address is saved in routing table of AODV whereas active precursor node and next active node IP address is entered in routing table of RDCLRP-FUZZY.

- Surrounding nodes save active nodes IP address in RDCLRP-FUZZY.

- The critical node warns about the route failure possibility in RDCLRP-FUZZY. There is no such provision in AODV.

- In AODV, route is repaired locally if route failure is near to destination. But if route fails in between route then a notice about route failure is given to the source node. This reinitiate the process of route discovery. In RDCRP, the route is repaired locally in advance before route failure.

- In RDCLRP, route failure is predicted. When the node traverses the threshold range of speed or received signal strength (RSS) or residual battery power (RBp) then that node becomes critical node and shows possibility of route break through admonition.

- Periodic HM for route maintenance and aperiodic HM for network connectivity is used in AODV. In RDCLRP-FUZZY, HM is used only for route maintenance. This reduces flooding and hence battery power consumption of inactive nodes. When these nodes will become active nodes later, they can utilize unused battery power and thus the possibility of route breakdown is reduced.

- In AODV, route failure rate is high whereas in RDCLRP-Fuzzy, it is reduced.

The route is searched through flooding of RREQ. If a node has route or is destination itself then it sends RREP. If a node receives RREP, the route is established. The established path is called as active path and nodes participating in data communication are called active nodes. All active nodes call speed, RSS, RBp value at the network layer and this is the cross layer approach. 
If speed or RSS value or RBp traverses its threshold then that active node is said to be critical node. The critical node warns their neighbours about its critical situation thrugh broadcast of Hello warning message (HWM). HWM consists of addresses of precursor and next active node which are participating in the communication of data. Neighbor nodes listen the admonition message and they will see the address of active neighbor nodes of critical node in its routing table. If neighbor node have the addresses of both active neighbors in its routing table then it will reply to critical node through Hello warning reply message (HWR). The node which replies first will participate in new route. The critical node broadcast service replicate message (SRM) to the active neighbours of critical node and to new node from whom HWR arrived. The SRM consist of new node id and active neighbor node id. Thus the critical node will be replaced by new node before link failure. So a new route is discovered through HWM. Critical node searches the route locally and tries to do preemptive route repair.

\section{HI Calculation:}

Route failure depends on the value of residual battery power (RBp), speed (S) and direction of a node i.e. received signal strength (RSS). Therefore HWM is made adaptive.

The HWM is broadcast after an interval called HWM interval (HI). This interval is decided on the basis of Fuzzy logic. Fuzzy logic is a tool for mapping the input features to the output based on data in the form of "IF - Then" rules controller $[15,16]$. Here three input features are taken, namely RSS, RBp and S. The key data for the system model is listed below in Tab. 1. We found that Mamdani model is applicable for our purpose, which evaluates the final output. For implementation, MATLAB Fuzzy inference engine is being used. Fuzzified input data trigger one or several rules in the Fuzzy model to calculate the result [17]. In this paper 27 rules are mapped. The rule table for calculation of $\mathrm{HI}$ is given in $[18,19]$.

The output (HI) ranges are taken from 0 to $12 \mathrm{sec}$ in proposed work. Simplifying, the signal strength from 0 to $-90 \mathrm{dbm}$ (low $=-90 \mathrm{dbm}$ to $-80 \mathrm{dbm}$, medium $=-80 \mathrm{dbm}$ to $-70 \mathrm{dbm}$, high $=-70 \mathrm{dbm}$ to $-60 \mathrm{dbm}$ ) are taken [19]. This gives a very low potential value for all signal strength values. We use the speed from $0 \mathrm{mps}$ to $20 \mathrm{mps}$ and residual battery power from $0.2 \mathrm{mAhr}$ to $0.8 \mathrm{mAhr}$. The rules are written in Table 1 . The selected ranges for $\mathrm{L}, \mathrm{M}$ and $\mathrm{H}$ are given in Table 2. Table 3 shows calculated values of $\mathrm{HI}$ for $\mathrm{RBp}=\mathrm{H}$ by Fuzzy inference engine. Table 4 and Table 5 provide values of $\mathrm{HI}$ for $\mathrm{RBp}=\mathrm{M}$ and $\mathrm{RBp}=\mathrm{L}$ respectively.

Table 1 Rule table for calculation of $\mathrm{H}^{-}$

\begin{tabular}{|c|c|c|c|c|c|c|c|c|c|}
\hline \multirow{2}{*}{ S } & \multicolumn{3}{|c|}{ Low(L) } & \multicolumn{3}{c|}{ Medimm(M) } & \multicolumn{3}{c|}{ High(H) } \\
\cline { 2 - 11 } & \multicolumn{3}{|c|}{ RSS } & \multicolumn{3}{c|}{ RSS } & \multicolumn{3}{c|}{ RSS } \\
\cline { 2 - 11 } & L & M & H & L & M & H & L & M & H \\
\hline L & L & L & L & H & M & H & H & H & H \\
\hline M & L & L & L & M & M & H & M & M & H \\
\hline H & L & L & L & L & L & M & L & L & M \\
\hline
\end{tabular}

Table 2 Range table for calculation of He

\begin{tabular}{|c|c|c|c|c|}
\hline Range & $\begin{array}{l}\text { Speed } \\
\text { (Inps) }\end{array}$ & $\begin{array}{l}\text { Battery } \\
\text { Power } \\
\text { (nAhr) }\end{array}$ & $\begin{array}{l}\text { Recrived } \\
\text { signal } \\
\text { strength } \\
\text { (-dbin) }\end{array}$ & $\underset{\text { (s) }}{\text { HI }}$ \\
\hline $\operatorname{Lw}(L)$ & 8-12 & $02-0.4$ & $(-90)-(-80)$ & $0-4$ \\
\hline $\begin{array}{c}\text { Medinm } \\
\text { (M) }\end{array}$ & $12-16$ & 0.40 .6 & $(-80)-(-70)$ & 48 \\
\hline High(H) & $16-20$ & $0.6-0.8$ & $(-70)-(-60)$ & $8-12$ \\
\hline
\end{tabular}




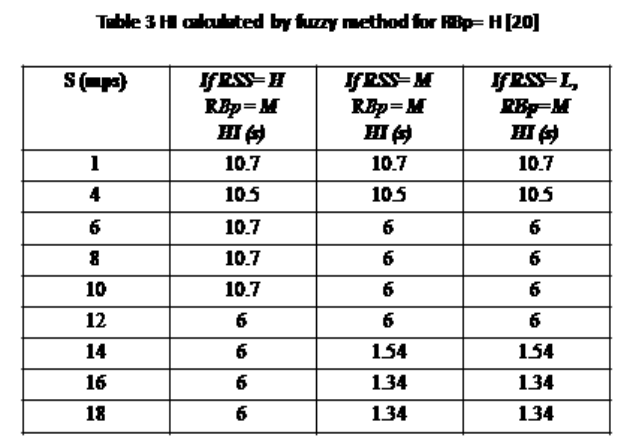

\begin{tabular}{|c|c|c|c|}
\hline$S([p)$ & $\begin{array}{c}f R S=H, \\
W_{P}=H \\
H_{(A)}\end{array}$ & 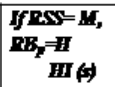 & $\begin{array}{c}\text { JfRSS }=L_{,}, E_{2}= \\
H_{(A)}\end{array}$ \\
\hline 2 & 10.7 & 6 & 10.7 \\
\hline 4 & 105 & 6 & 105 \\
\hline 6 & 10.7 & 6 & 6 \\
\hline 8 & 10.7 & 6 & 6 \\
\hline 10 & 10.7 & 6 & 6 \\
\hline 12 & 6 & 6 & 6 \\
\hline 14 & 6 & 154 & 154 \\
\hline 16 & 6 & 121 & 134 \\
\hline 18 & 6 & 1.13 & 134 \\
\hline
\end{tabular}

\begin{tabular}{|c|c|c|c|}
\hline $\mathbf{S}_{(\sim p)}$ & 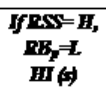 & $\begin{array}{c}\text { JfRS }=M, \\
\text { RB, }= \\
\text { HI }\end{array}$ & 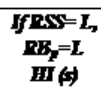 \\
\hline 2 & 134 & 134 & 134 \\
\hline 4 & 154 & 154 & 154 \\
\hline 6 & 154 & 154 & 154 \\
\hline 8 & 134 & 134 & 134 \\
\hline 10 & 134 & 134 & 134 \\
\hline 12 & 134 & 134 & 134 \\
\hline 14 & 154 & 154 & 154 \\
\hline 16 & 134 & 134 & 134 \\
\hline 18 & 134 & 134 & 134 \\
\hline
\end{tabular}

\section{Parameters Calculation}

\section{Speed calculation:}

The coordinates of the nodes are maintained at last known coordinates at time $\left(\mathrm{t}_{1}\right)$ and present coordinates at time $\left(\mathrm{t}_{2}\right)$ to calculate speed

Speed $=$

$$
\frac{\sqrt{\left(\mathrm{x}_{2-} \mathrm{x}_{1}\right)^{2}}+\sqrt{\left(\mathrm{y}_{2-} \mathrm{y}_{1}\right)^{2}}}{\mathrm{t}_{2-} \mathrm{t}_{1}}
$$

This formula is used for calculation of speed.

\section{RSS Calculation:}

The Free-space propagation model relation is given in equ. 1 and used for calculation of received power.

$\mathrm{P}_{\mathrm{R}}=\mathrm{P}_{\mathrm{T}}\left[\frac{\lambda}{4 \pi \mathrm{d}}\right]^{2} \mathrm{G}_{\mathrm{TG}_{\mathrm{R}}}$

where $\lambda$ is wavelength of carrier, $d$ is distance between sender and receiver, $G_{T}$ and $G_{R}$ are gain of transmitting and receiving antennas respectively. The effects of noise and fading are not considered [21]. This received power is taken as RSS.

\section{Residual Battery Power (RBp) Calculation:}

The battery power consumption (BPC) is calculated as given in equ. 2 . $B P C=\left(N_{t} * I * P_{t}\right)+\left(N_{r} * J * P_{r}\right)+\left(P_{\text {idel }}\right)+\left(P_{\text {sleep }}\right)$ 
Where $\mathrm{N}_{\mathrm{t}}$ is number of times packet transmitted, $\mathrm{I}$ is size of transmitted packet, $\mathrm{P}_{\mathrm{t}}$ is battery power consumption in transmission of a packet, $\mathrm{N}_{\mathrm{r}}$ is number of times packet is received, $\mathrm{J}$ is size of received packet, $\mathrm{P}_{\mathrm{r}}$ is battery power consumption in reception of a packet, $P_{\text {idel }}$ is battery power consumption in ideal mode, $P_{\text {sleep }}$ is battery power consumption in sleeping mode [22, 23].

The residual battery power(RBp) can be calculated as given below

$R B p=$ Maximum battery power $-B P C$

Formula used for Defuzzification:

$$
f=\frac{\sum_{i=1}^{p} x_{i} * \mu\left(x_{i}\right)}{\sum_{i=1}^{p} \mu\left(x_{i}\right)}
$$

Equ.4 shows the defuzzification functions for $\mathrm{f}$. The defuzzification method uses the centroid, where $\mathrm{x}_{\mathrm{i}}$ is the input, $\mathrm{p}$ is the number of rules, and $\mathrm{f}$ is the output [17].

\section{Results and Analysis}

In the following experiments, two aspects of proposed scheme were investigated. The impact of traffic and mobility are tested by varying the total number of sent packets and by varying pause time respectively. Simulation of the protocol is done on the qualnet simulator 5.0. Performance of proposed scheme is compared with basic AODV protocol. Following are the scenario parameters were constant throughout the simulation, written in Table 5.

Table 5 connen scenari paraneters

\begin{tabular}{|c|c|}
\hline \multicolumn{2}{|c|}{ Connen Scenari paraneters } \\
\hline No. of nodes & 100 \\
\hline Packetsize & 512 bytres \\
\hline Simulation Time & 900sec \\
\hline Chrmel & Path loss modd \\
\hline Energy modd & Micaz \\
\hline Battrey modd & Limex \\
\hline Mobility modd & Random way point \\
\hline Strit of trmsmision & Aftri $1 \mathrm{sec}$. \\
\hline Fnd of trmsmissian & $900 \mathrm{sec}$ \\
\hline Datn rate & 2Mbs \\
\hline Node trmsmissionrmge & $399 \mathrm{~m}$ \\
\hline Simulation aren & $1500 \mathrm{~m} \times 1500 \mathrm{~m}$ \\
\hline
\end{tabular}

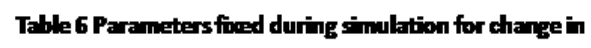

pausetine and traffic

\begin{tabular}{|c|c|c|}
\hline Paraneter & $\begin{array}{c}\text { Varintion in pause } \\
\text { tiine }\end{array}$ & $\begin{array}{c}\text { Varintion in tht } \\
\text { number of } \\
\text { parkets sent }\end{array}$ \\
\hline Panse time & 100 sec to 500 sec & 50 sec \\
\hline Limk & 20 & 20 \\
\hline Speed & $20 \mathrm{mps}$ & $20 \mathrm{mps}$ \\
\hline $\begin{array}{c}\text { Totrl packet } \\
\text { sent }\end{array}$ & $\begin{array}{l}899 \text { packetsin } \\
\text { simulationtime }\end{array}$ & $\begin{array}{c}899 \text { packetsto } 4495 \\
\text { packetsin } \\
\text { simulationtime }\end{array}$ \\
\hline
\end{tabular}

Results shown in figures from 1 to 7 have been analyzed for variation in pause time to check the impact of mobility and traffic. The variable parameters are written in Table 6 which are used during simulaton. Throughput, end to end delay, packet delivery ratio, average jitter, number of times link failure, residual battery power and number of times HM sent during simulation time have been analyzed for proposed scheme. 


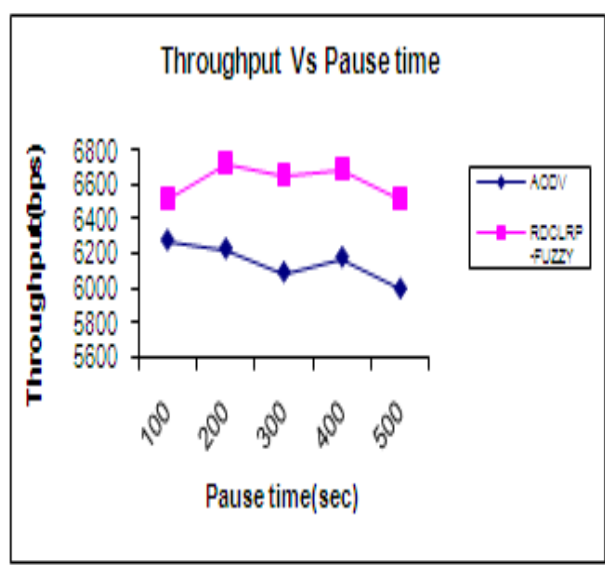

Figure 1. Throughput with Variation In (A) Pause Time

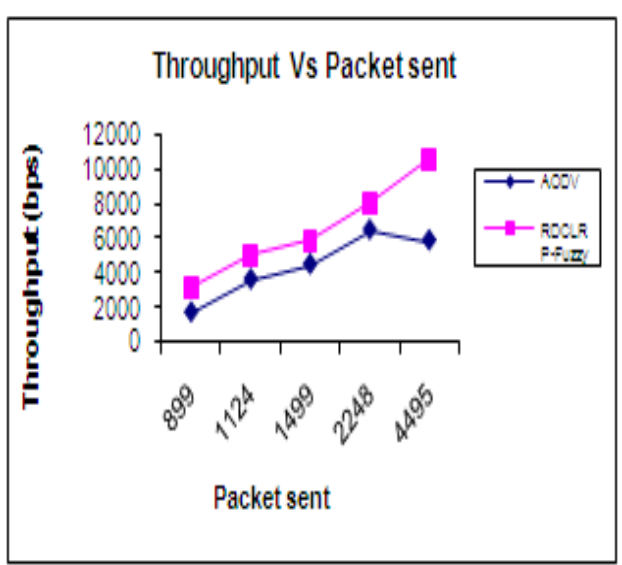

Figure 1 Throughput With Variation in (B) Total Number of Sent Packets

The mobility increases when pause time reduces resulting in increases the chances of change in topology. This degrades the performance of the network like decrease in throughput and total number of received packets. With the increase in total number of sent packets, traffic increases. This increases the possibility of congestion. If congestion increases, packets may drop resulting in less reception of data, ultimately this decreases throughput. But if congestion is less, throughput will be more. RDCLRP-FUZZY shows better performance due to availability of alternate route before route failure in comparison of AODV as shown in Figure 1 (a) and (b), it is because of transmission of less number of control packets and replacement of critical node with available neighbor node, switching of route before route break. Similarly the number of received packets is highest in RDCLRP- FUZZY as shown in Figures 4 (a) and (b).

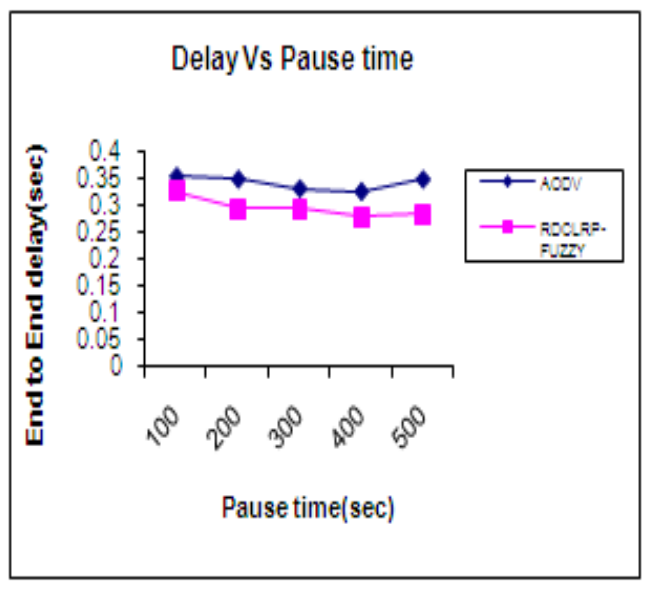

Figure 2. End to End Delay With Variation in (A) Pause Time

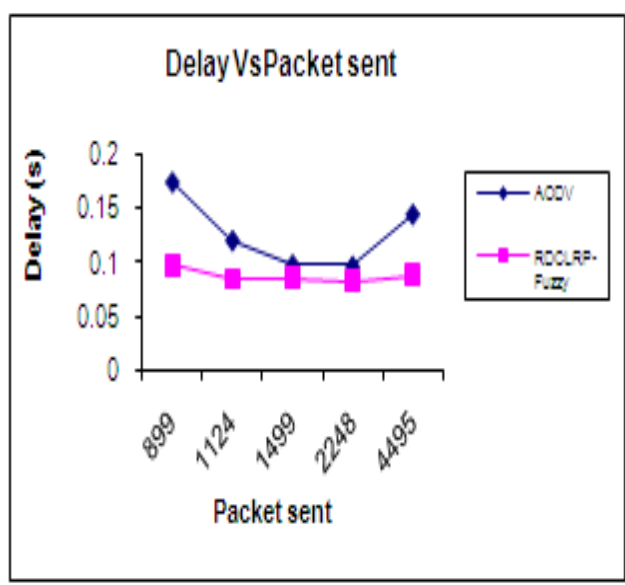

\section{Figure 2 End to End Delay with Variation In (A) Total Number of Sent Packets}

The end-to-end average delay indicates the time taken in the transmission of data from source node to destination. The end to end delay should be less for better performance. The increase in mobility or traffic increases the chances of route breakage. In the case of AODV protocol, if route breaks near the destination, local route repair method is used. But if route error occurs at any other place in between the route, RERR message is generated by the node detecting the link break to the source node. Then the source node reinitiates the path discovery process. This increases control traffic in the network. If 
route breaks frequently, the situation will be exploding. So the packets would take more time to reach to destination results in more delay as shown in Figures 2 (a) and (b). RDCLRP-FUZZY reduces possibility of route breakage and also reduces propagation of control packets. That's why, RDCLRP-FUZZY shows less delay and better performance than the AODV.

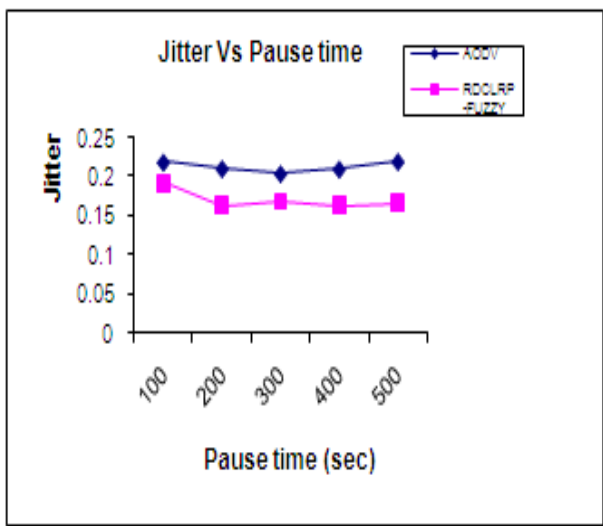

Figure 3. Jitter with Variation In (A) Pause Time

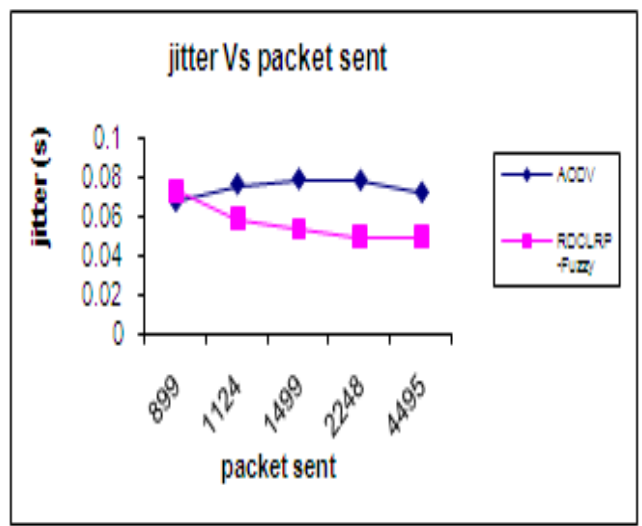

Figure 3. Jitter with Variation In (A) Total Number Of Sent Packets

Jitter is the time taken by two packets reached to a node, caused by network congestion, queue, route switching. It should be less for a routing protocol to perform better. The increased mobility could increase the route switching. The traffic increases if the route switching is more. Congestion increases with the increase in traffic and packet may go into the queue. This causes delay and jitter. The average jitter is shown in Figure 3 (a) and (b). The RDCLRP-FUZZY is performed better for mobility and with the increase in traffic than AODV as route change is less in proposed algorithm.

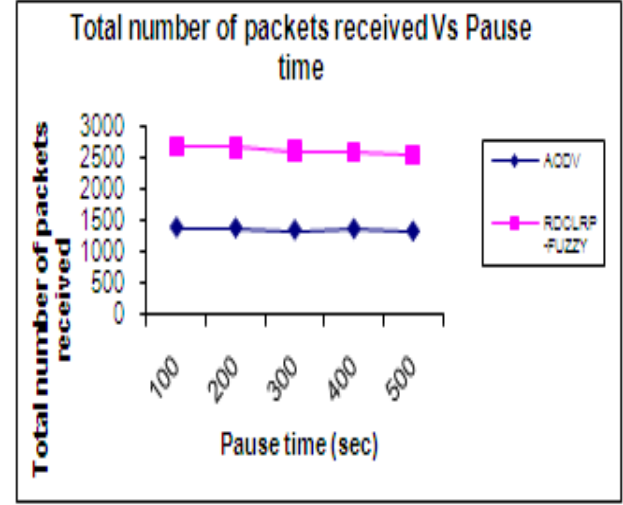

Figure 4. Number of Received Packets with Variation in (A) Pause Time

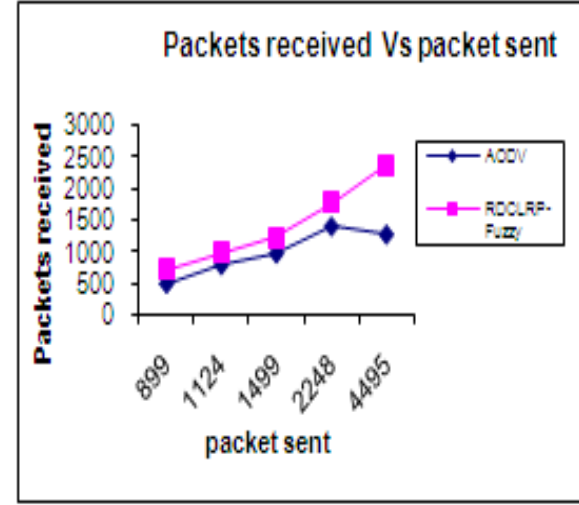

Figure 4. Number of Received Packets with Variation in (B) Total Number Of Sent Packets

In case of high mobility and traffic, no. of received packets will definitely be more. Thus, some nodes may run out of battery or go out of transmission range during the simulation and hence they could not forward packets any more. This can be one of the reason of sending less number of packets in AODV. But in proposed algorithm, the bad status route is replaced by alternate route before route break, so the nodes are able to forward packets for a long period of time. Thus the number of received packets are more for proposed scheme in comparison of AODV as shown in Figure 4 (a) and (b). 


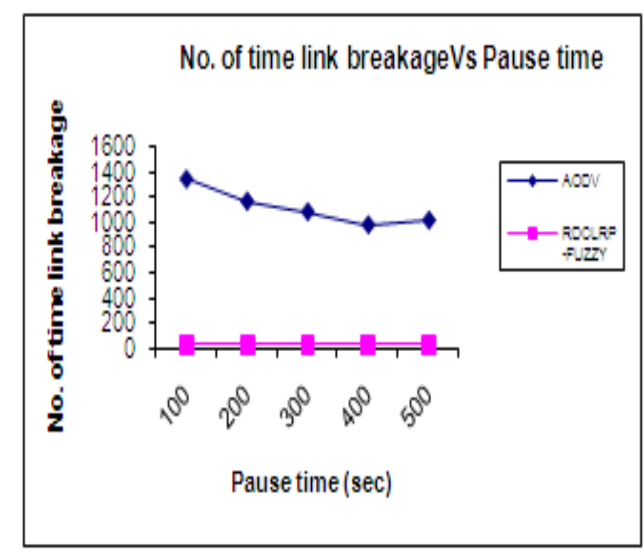

Figure 5. Number of Times Link Breakage with Variation in (a) Pause Time

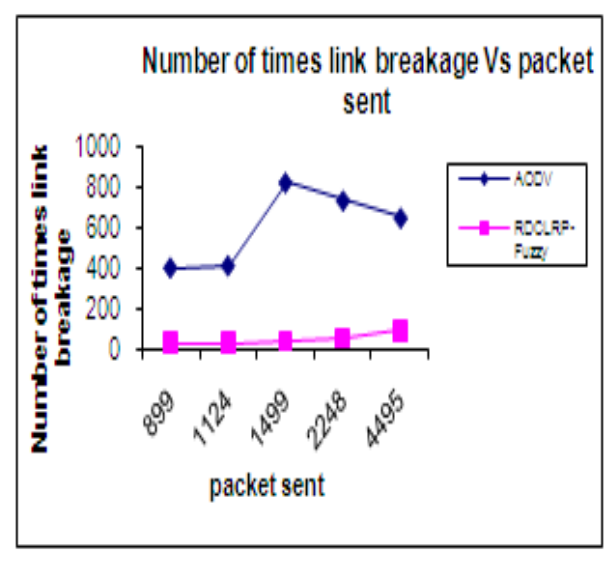

Figure 5. Number of Times Link Breakage with Variation in (b) Total Number of Sent Packets

Links may break during the transmission of data due of movement of intermediate nodes or may be because of power failure of intermediate nodes. More traffic and high mobility results in frequent link breakage inturn results in the transmission of more control packets for route repair in AODV that's why the number of times link breakage is more and residual battery power is less in AODV as shown in Figures 5 (a), (b) and 6 (a), (b) respectively.

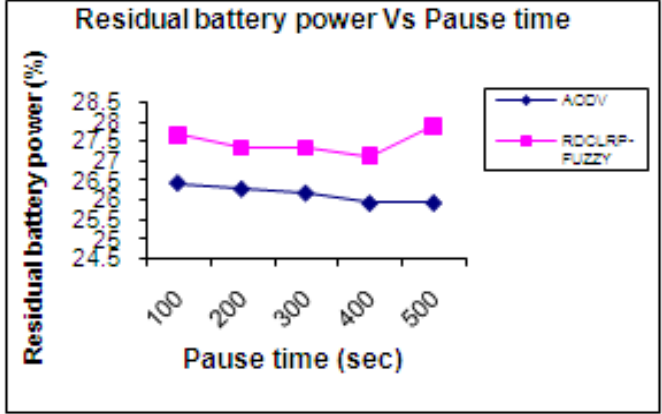

Figure 6. Residual Battery Power with Variation in (b) Pause Time

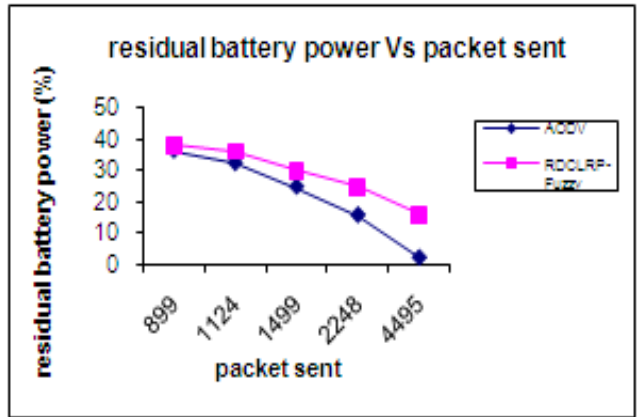

Figure 6. Residual Battery Power with Variation in (b) Total Number of Sent Packets

The AODV uses HM for network connectivity [13] and send periodically by active nodes for route maintenance. This increases control traffic unnecessary, drains the battery power quickly resulting in route break. The RDCLRP-FUZZY does not send HM for network connectivity. Only active nodes send HM for route maintenance untill node does not become critical. If node comes in critical situation then that node sends HM as HWM. Finally route is repaired using HWM, HWR and SRM before route break. Thus number of HM sent is less in RDCLRP-FUZZY in comparison of basic AODV as shown in Figure 7 (a) and (b). This reduces control traffic. 


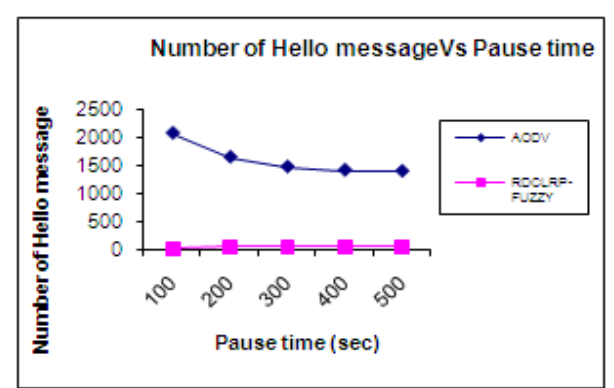

Figure 7. Total Number of Hello Messages Sent With Variation In (A) Pause Time

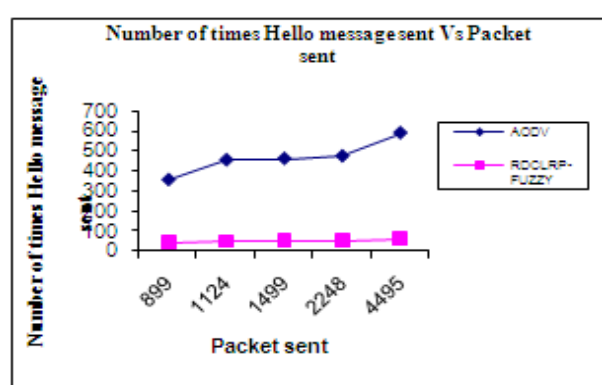

Figure 7. Total Number Of Sent Packets of Hello Messages Sent With Variation In (B) Total Number of Sent Packets

The RDCLRP-FUZZY has improved in all performance parameters over AODV. Number of times link breakage is minimized, so residual battery power is increased, in turn end to end delay and jitter reduces which increases throughput. The number of times HM sent is reduced remarkably, this reduces unnecessary overhead of the network. So the RDCLRP- FUZZY route repair scheme is better than basic AODV route repair mechanism.

\section{Conclusion}

RDCLRP-FUZZY is introduced as an improved algorithm based on preemptive route repair concept using cross layer approach and fuzzy logic. The route maintenanace scheme of AODV is modified. RDCLRP-FUZZY keeps route alive for long time due to admonition about possibility of route failure. If an active route is in critical condition then that route is switched to an alternate route in advance before route failure. The performance of this algorithm is analyzed and compared with basic AODV to test for the variation of mobility and traffic keeping constant number of nodes and terrain area. It is observed that the proposed scheme improves the performance of the network. It is calculated that the route failure is reduced by an average of $91 \%$. The number of sent HM is also reduced in proposed scheme. This also improves other performance parameters like throughput, delay, jitter, number of received packets. Thus it can be used for real time and non real time communication.

\section{References}

[1] M. Fotino and F. De Rango, "Energy Issues and Energy aware Routing in Wireless Ad-hoc Networks", International Journal of Communication System, Mobile Ad-Hoc Networks: Protocol Design, (2010), pp. 281-296.

[2] A. Kumar, M. Q. Rafiq and K. Bansal, "Energy Efficient Routing Protocol Avoiding Route Breaks based on DSR", International Journal of Computer Applications (0975-8887), vol. 44, no. 4, (2012) April, pp. 39-44.

[3] S. Iyer, S. Bhattacharyya, N. Taft, N. McKeoen and C. Diot, "A measurement based study of load balancing in an IP backbone", SprintATL, Tech.Rep.TR02-ATL-051027, (2002) May.

[4] X. Huang and Y. Fang, "Performance Study of Node-Disjoint Multi-path Routing in Vehicular MANETs Networks", IEEE Transactions on Vehicular Technology, vol. 58, (2009) May, pp. 19421950.

[5] M. A. Razzaque, S. Dobson and P. Nixon, "Cross-Layer Self Routing: a self-managed routing approach for MANETs", IEEE, Computer Science, (2008).

[6] R. Winter, J. H. Schiller, N. Nikaein and C. Bonnet, "CrossTalk: Cross-Layer Decision Support Based on Global Knowledge”, IEEE Communications Magazine, (2006) January, pp. 93-99.

[7] T. Goff, N. B. Abu-Ghazaleh and D. S. Phatak, "Preemptive routing in ad hoc networks", Proc. of ACM SIGMOBILE, (2001), pp. 43-52. 
[8] W.-H. Yang, Y. Chiun Wang, Y.-C. Tseng and B.-S. P. Lin, "Energy-Efficient Network Selection with Mobility Pattern Awareness in an Integrated WiMAX and WiFi Network", s.l. : International journal of communication system, vol. 23, no. 2, (2010) February, pp. 213-230.

[9] X. Wu, G. Ding and W. Zhu, "Load-Based Route Discovery Through Searching Range Adaptation for MANET throughput Improvement", IEEE Transactions on Vehicular Technology, vol. 58, no. 4, (2009) May, pp. 2055-2066.

[10] C. E. Perkins and E. M. Royer, "Ad hoc on-demand distance vector routing", Proceedings of the $2^{\text {nd }}$ IEEE Workshop on Mobile Computing Systems and Applications, (1999) February, pp. 90-100.

[11] E. M. Royer and C. E. Perkins, "An Implementation Study of the AODV Routing Protocol", Proceedings of the IEEE Wireless Communications and Networking Conference, Chicago, (2000) September.

[12] C. E. Perkins, "Performance comparison of two on-demand routing protocols for ad hoc networks", IEEE Personal Communications, (2001), pp. 16-28.

[13] I. D. Chakeres and E. M. Belding-Royer, "The Utility of HMs for determining Link Connectivity", International Symposium on Wireless Personal Multimedia Communications - WPMC, vol. 2, (2002), pp. 504-508.

[14] C. Perkins, E. Belding-Royer and S. Das, "Ad hoc On-Demand Distance Vector (AODV) Routing", Request for Comments, vol. 3561, (2003) July.

[15] A. Lekova, K. S. Skjelsvik, V. Goebel and T. Plagemann, "Fuzzy Logic-Based Event Notification in Sparse MANET", Advanced Information Networking and Applications Workshops, AINAW '07. 21st International Conference, IEEE Computer Society, (2007), pp. 296-301.

[16] Guenter Prochart and Reinhold Weiss Reiner Schmid and Gerald Kaefer, "Fuzzy-based Support for Service Composition in Mobile Ad Hoc Networks", IEEE International Conference on Pervasive Services, 15-20 July 2007, pp:379 - 384.

[17] C. Lee, "Fuzzy Logic In Control Systems: Fuzzy Logic Controller-Part I", IEEE Transactions on Systems, Man, and Cybernetics, Vol. 20, No. 2, 1990, pp. 404-418.

[18] M. Fatima, R. Gupta and T. K. Bandhopadhyay, "RDCLRP-Route Discovery by Cross Layer Routing Protocol for Performance Improvement", Kanyakumari, India. s.1. : International Conference on Network and Computer Science, vol. V5-306, (2011) April.

[19] "RDCLRP-Route Discovery by Cross Layer Routing Protocol for Manet Using Fuzzy Logic Information Technology and Mobile Communication", Communications in Computer and Information Science, vol. 147, (2011), pp. 348-351.

[20] M. Fatima, R. Gupta and T. K. Bandhopadhyay, "Route Discovery by Cross Layer Approach for MANET", s.l. : International Journal of Computer Applications, vol. 37, no. 7, (2012) January, pp. 0975-8887.

[21] M. Ramakrishnan, S. B. M. Priya and S. Shanmugavel, "Mathematical Modeling of Routing Protocol Selection for Optimal Performance of MANET", Second International Conference on Computer and Network Technology, IEEE, (2010).

[22] S. Foster, C. Calwell, T. Reeder and R. Neugebauer, "Battery Chargers and Energy Efficiency: Summary of Findings and Recommendations", Natural Resources Defense Council, (2003) August.

[23] C.-K. Toh, "Maximum Battery Life Routing to Support Ubiquitous Mobile Computing in Wireless Ad hoc Networks", IEEE Communications Magazine, vol. 39, no. 6, (2001) June, pp. 138-147. 
International Journal of Future Generation Communication and Networking Vol.6, No.6 (2013) 